\title{
BMJ Open Safety and efficacy of tocotrienol supplementation for bone health in postmenopausal women: protocol for a dose-response double-blinded placebo-controlled randomised trial
}

\author{
Chwan-Li Shen, ${ }^{1}$ Huanbiao Mo, ${ }^{2}$ Shengping Yang, ${ }^{1}$ Shu Wang, ${ }^{3}$ Carol K Felton, ${ }^{4}$ \\ Michael D Tomison, ${ }^{1}$ Ima Nirwana Soelaiman ${ }^{5}$
}

To cite: Shen C-L, Mo H, Yang S, et al. Safety and efficacy of tocotrienol supplementation for bone health in postmenopausal women: protocol for a doseresponse double-blinded placebo-controlled randomised trial. BMJ Open 2016;6:e12572.

doi:10.1136/bmjopen-2016012572

- Prepublication history for this paper is available online. To view these files please visit the journal online (http://dx.doi.org/10.1136/ bmjopen-2016-012572).

Received 9 May 2016 Revised 23 September 2016 Accepted 26 October 2016

CrossMark

For numbered affiliations see end of article.

Correspondence to Dr Chwan-Li Shen; Leslie.Shen@ttuhsc.edu

\begin{abstract}
Introduction: Osteoporosis is a major health concern in postmenopausal women, and oxidative stress contributes to the development of bone loss. Cellular studies and ovariectomised rat model mimicking bone loss in postmenopausal women show the boneprotective effect of tocotrienols (TTs) with antioxidant capability. We aim to access the safety and efficacy of TT consumption for bone health in postmenopausal women.
\end{abstract}

Methods and analysis: In this 12-week randomised double-blinded placebo-controlled trial for the effects of dietary TT supplementation in postmenopausal women, postmenopausal women aged 45 years and older with at least 1 year after menopause and bone mineral density T-score at the spine and/or hip 2.5 or more below the reference values will be randomly assigned to 3 daily supplements: (1) placebo group receiving $860 \mathrm{mg}$ olive oil, (2) low TT group receiving $430 \mathrm{mg}$ of $70 \%$ pure TTs (containing $300 \mathrm{mg} \mathrm{TT}$ ) and (3) high TT group receiving $860 \mathrm{mg}$ of $70 \%$ pure TTs (600 mg TT). The primary outcome measure will be urinary $\mathrm{N}$-terminal telopeptide. The secondary outcome measures will be serum bone-specific alkaline phosphatase, receptor activator of nuclear factor- $\mathrm{\kappa} B$ ligand, osteoprotegerin, urinary 8-hydroxy-2'deoxyguanosine and quality of life. At 0, 6 and 12 weeks, the following will be assessed: (1) primary and secondary outcome measures; (2) serum TT and tocopherol concentrations; (3) physical activity and food frequency questionnaires. Liver function will be monitored every 6 weeks for safety. 'Intent-to-treat' principle will be employed for data analysis. A model of repeated measurements with random effect error terms will be applied. Analysis of covariance, $\chi^{2}$ analysis and regression will be used for comparisons.

Ethics and dissemination: This study was approved by the Bioethics Committee of the Texas Tech University Health Sciences Center. The findings of this trial will be submitted to a peer-reviewed journal in the areas of bone or nutrition and international conferences.

Trial registration number: NCT02058420; results.

\section{Strengths and limitations of this study}

- This randomised, double-blind, placebocontrolled trial will help to assess the safety and efficacy of dietary tocotrienol supplementation on bone health in postmenopausal women who are at high risk of osteoporosis.

- This study will be performed at a research centre with experience in conducting independent, investigator-initiated, randomised controlled trials in nutrition and bone research.

- This study is a single-centre study. Tocotrienol supplement is not available over the counter worldwide.

- There is no long-term follow-up.

\section{BACKGROUND}

Reduced bone mass, microstructural deterioration, increased bone fragility and higher susceptibility to fractures including those associated with hip, spine and wrist ${ }^{1}$ are some of the characteristics of osteoporosis, a degenerative bone condition that is four times more likely found in women than in men. The gender disparity is attributed to postmenopausal decrease in oestrogen level and lighter and thinner bones in women in general. $^{2}$ Over half of postmenopausal women will experience a bone fracture as the result of osteoporosis. ${ }^{2}$ Using the WHO definition, ${ }^{3}$ the National Osteoporosis Foundation has estimated that at present, $\sim 8$ million women in the USA have osteoporosis, and an additional 22 million are estimated to have osteopenia that places them at increased risk for osteoporosis. ${ }^{4}$ Therefore, preventing and decelerating the development of osteoporosis in postmenopausal women has become a major public health strategy. 
Clinical application of bone densitometry, such as dual-energy X-ray absorptiometry, is generally used to measure bone mineral density (BMD) at the spine, hip and femoral neck to predict the fracture risk, to monitor the natural progression of diseases that affect BMD, or to monitor the therapeutic response to osteoporosisspecific treatments 1-2 years after inception of therapies. $^{5-8}$ In addition to BMD, serum bone turnover markers (bone formation markers and bone resorption markers) can offer another indication of the effectiveness of certain therapies ${ }^{9}$ including dietary supplements for restoring BMD. ${ }^{10} 11$ Bone turnover markers have shown to be promising in predicting fractures in the elderly up to 2 years before the event. ${ }^{12-14}$ A higher level of bone resorption rate is significantly associated with faster BMD loss. ${ }^{12-14}$ Decreased vertebral BMD and increased bone turnover have approximately equal power to predict the risk of bone loss and osteoporosisrelated fracture rate in postmenopausal women. ${ }^{12-14}$ Therefore, efforts to decrease the occurrence of dilapidation fractures should concentrate on postmenopausal women diagnosed with low BMD. Since bone turnover markers have been increasingly recognised as important for bone metabolism, using bone turnover markers should be the first step to monitor the early response of intervention in this population.

Studies have shown bone loss can be attributed to dysregulation of osteoblast and osteoclast activity mediated by increased oxidative stress through the ageing process. ${ }^{15} 16$ The benefits of antioxidant supplements such as tocopherols (TPs) or tocotrienols (TTs; two major isoforms of vitamin E) to bone health have been shown in cellular ${ }^{17-20}$ and animal studies, ${ }^{21-33}$ including those using the ovariectomised rat model mimicking bone loss in postmenopausal women. In cellular and animal studies, TTs are much more beneficial than TPs in bone protection. Cohort observational studies in humans, however, have shown positive, ${ }^{34-37}$ negative $^{38}$ or lack of ${ }^{39}$ associations between TP intake/serum TP concentration and BMD, casting doubts on the benefits of TPs. ${ }^{40}$ No cohort observational studies or randomised clinical trials have been conducted to address the effect of TTs on bone health in humans, especially in postmenopausal women who are at high risk for bone fracture. Therefore, the objective of this study is to evaluate the safety and efficacy of dietary TT supplements on bone health-related markers and mechanisms in postmenopausal women. We hypothesise that 12 weeks of TT supplements would benefit bone remodelling, as measured by bone markers in postmenopausal women with normal bone status and osteopenia, compared with those receiving placebo only, and the changes in bone markers associated with bone remodelling would be correlated with changes in oxidative stress. This hypothesis is based on the premise that TTs may reduce oxidative stress that adversely affects bone health by increasing differentiation and function of osteoclasts ${ }^{41}$ and inhibiting osteoblastic differentiation. $^{42}$
In this paper, we present the design and detailed protocol of a double-blinded placebo-controlled and randomised trial, as well as a discussion of the overall challenges of conducting this trial. The results from this trial will be reported at the completion of the study in accordance with the Consolidation of Standards for Reporting Trials guidelines. ${ }^{43}$

\section{METHODS/DESIGN}

\section{Study design}

This is a 12-week double-blinded placebo-controlled and randomised intervention trial with allocation 1:1:1 for three treatment arms. Women 45 years and older with at least 1 year after menopause but no osteoporosis will be recruited primarily from local independent senior facilities, municipal senior community centres, and obstetrics and gynaecology clinics. A final sample size of 22 participants per group with an expected attrition rate of $15 \%$ over 12 weeks of intervention will produce an initial sample size of 78 participants. This sample size will yield a power of $\sim 0.80$ at $\alpha=0.05$ for detecting differences in primary outcome (eg, urinary NTX). After screening, qualified participants will be matched for age and randomly assigned to one of the three treatment groups: placebo, low TT and high TT. During the 12-week intervention, all participants will be provided with $500 \mathrm{mg}$ elemental calcium and $400 \mathrm{IU}$ vitamin D daily. The participants in the placebo group will receive two $430 \mathrm{mg}$ olive oil softgels per day (one in the morning and another in the evening) for 12 weeks. The low TT participants will receive one $430 \mathrm{mg}$ olive oil softgel in the morning and one $430 \mathrm{mg}, 70 \%$ pure TT softgel (representing $300 \mathrm{mg}$ TT) in the evening for 12 weeks. The high TT participants will receive two $430 \mathrm{mg}$, $70 \%$ pure TT softgels per day (representing $600 \mathrm{mg}$ TT; one in the morning and another in the evening) for 12 weeks. The primary outcome measure is concentrations of urinary $N$-terminal telopeptide (NTX, a bone resorption marker) and the secondary outcome measures are the levels of serum bone-specific alkaline phosphatase (BAP, a bone formation marker), serum receptor activator of nuclear factor- $\mathrm{kB}$ ligand (sRANKL), serum osteoprotegerin (OPG), and urinary 8-hydroxy-2'-deoxyguanosine (8-OHdG, an oxidative stress DNA damage marker). Both primary and secondary outcome measures will be taken from the participants at baseline, 6 and 12 weeks. Liver function will also be monitored by assessing the activity of aspartate aminotransferase (AST) and alanine aminotransferase (ALT) at baseline, 6 and 12 weeks. The concentrations of serum TT will be determined at baseline, 6 and 12 weeks for bioavailability determination. Quality of life, physical activity and food frequency questionnaires will be assessed at baseline and 12 weeks. Investigators evaluating the endpoints will be blinded to interventions. The time points of all participant-related actions to be taken during the study period are presented in table 1 . 
Table 1 Timetable of activities planned during the course of the study directly related to participants

\begin{tabular}{|c|c|c|c|c|c|c|c|c|c|c|c|c|c|}
\hline \multicolumn{14}{|l|}{ Weeks } \\
\hline Activity & 0 & 2 & 4 & 6 & 8 & 10 & 12 & 14 & 16 & 18 & 20 & 22 & 24 \\
\hline Enrolment & $x$ & & & & & & & & & & & & \\
\hline Screening & $x$ & $x$ & & & & & & & & & & & \\
\hline Randomisation & & $x$ & & & $x$ & & & & & & & & \\
\hline Intervention & & $x$ & $x$ & $x$ & $x$ & $x$ & $x$ & $x$ & & & & & \\
\hline Anthropometry & $x$ & $x$ & & & $x$ & & & $x$ & & & & & \\
\hline Blood for outcomes & & $x$ & & & $x$ & & & $x$ & & & & & \\
\hline Liver function assessment & & $x$ & & & $x$ & & & $x$ & & & & & \\
\hline Pill counts & & $x$ & & & $x$ & & & $x$ & & & & & \\
\hline Food intake assessment & & $x$ & & & & & & $x$ & & & & & \\
\hline Physical activity survey & & $x$ & & & & & & $x$ & & & & & \\
\hline Quality of life survey & & $x$ & & & & & $x$ & $x$ & & & & & \\
\hline Self-report adverse event & $x$ & $x$ & $x$ & $x$ & $x$ & $x$ & $x$ & $x$ & & & & & \\
\hline
\end{tabular}

\section{Study setting, study population and recruitment}

The participants, women 45 years or older with at least 1-year postmenopausal history and being ambulatory, for this present study will be recruited from Lubbock, Texas and surrounding areas. Ethnicity or race is not a factor in the inclusion of participants. Direct person-to-person solicitation in the obstetrics/gynaecology clinic and health fairs, flyers, non-solicited email system, campus announcements, local radio, newspapers, senior newsletters, and TV scripts will be used to recruit potential participants. In addition, we plan to recruit the minority participants who have limited access to such intervention due to social and cultural factors. We will advertise and host information sessions, particularly at local minority community centres and churches, partnering with leaders identified within these organisations. Advertisement in minority newspaper and radio will also be implemented. Based on our experience, it is not difficult to recruit qualified participants within a reasonable time frame from our existing participant pool with the methods described above. If a longer period is needed to recruit the participants, the intervention may take place in a staggered fashion-a block randomisation strategy will place participants into subgroups for treatments on a first-come, first-served basis.

\section{Screening}

Prescreening will be conducted through a phone interview or medical record review and will cover menstrual history, medication for osteoporosis, age, availability for the study period and most recent BMD results if available. Participants who passed the prescreening will attend an informed consent session and sign consents and Health Insurance Portability and Accountability Act (HIPAA) forms before completing a detailed questionnaire with demographic, health and dietary information. Visits for BMD and fasting blood screenings will follow afterwards.

\section{Inclusion criteria}

1. Postmenopausal women with no menses for at least 1 year or a serum follicle-stimulating hormone $>30 \mathrm{IU} / \mathrm{mL}$.
2. Age 45 and older.

3. Bone mass with BMD T-score >-2.5 (nonosteoporotic) at measured site (spine and/or hip). ${ }^{3}$

4. Participants satisfying the above screening criteria will receive BMD measurement of the anteroposterior (AP) lumbar spine (L1-L4) and proximal femur (femoral neck, Ward's triangle and trochanter) by dual-energy X-ray absorptiometry scan (Norland Excell X-Ray Bone Densitometer, Serial No. 1490). Subject candidates must be diagnosed with no osteoporosis based on BMD T-score at the spine and/or hip $>-2.5$ below the young normal sex-matched areal BMD of the reference database. ${ }^{3}$ BMD $\left(\mathrm{gm} / \mathrm{cm}^{2}\right)$ will be determined by dividing bone mineral content (BMC; in grams of calcium hydroxyapatite) by the area $\left(\mathrm{cm}^{2}\right)$ of interest. Dividing BMC by area partially corrects for differences in bone size.

5. Normal function of thyroid, liver and kidney.

6. Serum 25 -hydroxy vitamin $\mathrm{D} \geq 20 \mathrm{ng} / \mathrm{mL}$.

7. No bisphosphonates at all if they had a treatment lasting at least 12 months.

\section{Exclusion criteria}

1. History of, or evidence for, metabolic bone disease including recent fractures (other than low BMD).

2. Having received medication (calcitonin, raloxifene or systemic glucocorticoids) within 3 months of the study initiation.

3. Having hormone/hormone-like replacement therapy within 6 months of the study initiation.

4. History of cancer within the past 5 years.

5. History or evidence of endocrine disease or malabsorption syndrome that would be a contraindication to the investigation of TT absorption.

6. Glycated haemoglobin of $\geq 7 \%$ in the past 3 months.

7. History of statin or other drugs for cholesterol control within 3 months of the study initiation.

8. Alcohol intake greater than 'moderate' (one drink per day) or use of non-steroidal anti-inflammatory drugs on a regular basis. 
9. Cognitive impairment, depression or other medical/eating disorders, likely to move during the trial, lack of transportation to the study site, or being unavailable at sample collection times.

10. Smoking $>10$ cigarettes/day.

11. Unwillingness to accept randomisation.

12. Taking anticoagulants that may interact with TTs.

\section{Sample size}

In this study, we plan to enrol 78 patients with 26 in each of the three arms. Since this is a pilot study to evaluate the effects of TT treatments on bone biomarkers, we use the primary outcome measures (urine NTX) for the sample size calculation. Using Power Analysis and Sample Size software (PASS V.11), a sample size of 22 in each of the placebo, low TT and high TT groups will achieve a power of $80 \%$ to detect a clinically significant difference between the group means of urinary NTX level at the end of 12 weeks. This calculation is based on an analysis of covariance (ANCOVA), adjusted for three covariates: baseline NTX, age and body mass index (BMI).

We will perform analysis of variance (ANOVA) analysis of all three groups. If the ANOVA result is significant, we will perform post hoc pair-wise comparison. Therefore, it is not necessary to adjust for multiple testing. PASS software is used to calculate the required sample size based on the assumptions: (1) NTX level change (primary outcome measure) has a mean of $0 \mathrm{nM}$ bone collagen equivalents (BCE)/mM creatinine (Crt) ng/ $\mathrm{mL}$ in the placebo group with a SD of $12.69 \mathrm{nM} \mathrm{BCE} /$ $\mathrm{mM} \mathrm{Crt.}{ }^{44}$ (2) Compared with the placebo group, the low TT and high TT groups will have a relative decrease of $15 \%$ and $20 \%$ from the baseline of $53.5 \mathrm{nM} \mathrm{BCE} /$ $\mathrm{mM}$ Crt, respectively. (3) We also assume that the adjusted covariates will explain about $20 \%$ of the variability in NTX, that is, a Pearson correlation coefficient of $0.45\left(R^{2}=0.20\right)$ between NTX and the adjusted covariates. Taking into consideration an expected attrition rate of $15 \%, n=26$ will be recruited for each group with a total of 78 participants to start the study.

\section{Randomisation and allocation concealment}

This study has a prospective, randomised, doubleblinded, placebo-controlled design. Randomisation is based on the computer-generated table of random digits. All participants who had passed the screening criteria will participate in randomisation where participants will be assigned to one of the three treatment arms with a fixed randomised scheme. Enrolled de-identified patients will be randomly assigned 1:1:1 to receive placebo, low TT and high TT by the study biostatistician. The allocation sequence is sealed in numbered and opaque envelopes to ensure that the sequence is concealed. In order to minimise the consequent bias, a stratified block randomisation method with varying block sizes of 3 and 6 will be employed to assign participants to one of the three groups: placebo, low TT and high TT groups. The strata will be constructed based on BMI $\left(\geq 30\right.$ or $\left.<30 \mathrm{~kg} / \mathrm{m}^{2}\right)$ and age $(\geq 50$ or $<50$ years $)$. We will provide de-identified codes of participants to the biostatistician who will randomise group assignment and generate a group assignment number for each participant.

TT and placebo with the same character are prepared by a pharmacist. Patients and all study personnel except the investigative pharmacist will be blind to treatment assignment. The details of the series are unknown to any of the study participants, coordinators and outcome assessors and are contained in a set of opaque, sealed and numbered envelopes.

\section{Intervention}

\section{Purchasing and blinding of study agents}

Placebo and TT of the same lot, respectively, will be provided by American River Nutrition (Hadley, Massachusetts, USA; Investigational New Drug (IND) number 120761 by the Food and Drug Administration (FDA)). Each placebo capsule of $430 \mathrm{mg}$ olive oil will contain no TT ingredient at detectable levels. Each TT capsule (DeltaGold Tocotrienol 70\%) will contain $430 \mathrm{mg}$ TT $(90 \% \quad \delta$-TT and $10 \% \quad \gamma$-TT) with a $70 \%$ purity, representing $300 \mathrm{mg}$ TT. The use of placebo to mask the control group is desirable in this study to keep respondents blinded to the TT treatment assignment. Placebo softgels will be made of the same size and colour as the TT softgels for identical appearance and taste. The placebo group will provide a comparison of blood and urinary outcome measures and serve as a basis to assess TT's effect.

\section{Provision of calcium plus vitamin D supplement}

The beneficial effects of dietary supplements and exercise on BMD depend on adequate $\mathrm{Ca}$ /vitamin $\mathrm{D}$ intake. ${ }^{45}$ According to the results of the National Health and Nutrition Examination Survey (NHANES; 19992002), ${ }^{46}$ the estimated average dietary calcium intake was $687 \pm 15 \mathrm{mg} /$ day in postmenopausal women, which was much below the recommended daily intake (1200 mg/day) for this population. Thus, we will provide calcium (500 mg elemental $\mathrm{Ca}$, as Oyster Shell)/ vitamin D (400 IU as cholecalcifero) supplement (GlaxoSmithKline) to ensure that recommended daily intake levels of calcium (1000-1200 mg) and vitamin D (600 IU) will be reached, taking into account their dietary intake and sun exposure. Supplementation of calcium and vitamin $\mathrm{D}$ has been employed in previous clinical trials to investigate the effects of short-term nutritional intervention on bone turnover markers and bone metabolism in postmenopausal women. ${ }^{11} 47$ We refrained from providing a higher dose of $\mathrm{Ca}$ /vitamin $\mathrm{D}$ to avoid blinding the TT effect by the strong antiresorptive activity of $\mathrm{Ca}$ /vitamin $\mathrm{D}$. 
Treatment arms

The study agent (TT, DeltaGold Annatto Tocotrienol) has generally recognised as safe (GRAS) status. Based on (1) TT fed at $60 \mathrm{mg} / \mathrm{kg}$ body weight in rats showed osteoprotective impacts, ${ }^{27} 2930$ and (2) the use of body surface area for dose translation from rat (250 $\mathrm{g}$ body weight) to human (70 kg body weight) ${ }^{48}$ the estimated effective dose of TT in humans for osteoprotection is $\sim 680 \mathrm{mg}$ daily. Therefore, we will test two dosages of TT (300 and $600 \mathrm{mg}$ ) for 12 weeks in this study. The current recommended dietary allowance (RDA) value of vitamin $\mathrm{E}, 15 \mathrm{mg} /$ day, for this study population is largely focused on $\alpha$-TP. No specific recommendation exists for TT. However, no adverse effects were observed with daily intake of $3.2 \mathrm{~g}$ or less $\mathrm{TT}^{49}$

Qualified participants will be randomly assigned into one of the three study groups (placebo, low TT and high TT). Participants in the placebo group will receive $860 \mathrm{mg}$ of olive oil daily ( $430 \mathrm{mg}$ olive oil softgel $\times 2$ per day, morning and evening after meal) for 12 weeks. Participants in low TT group will receive a $430 \mathrm{mg}$ olive oil softgel in the morning and a $430 \mathrm{mg}$ TT softgel in the evening daily for 12 weeks. Participants in high TT group will receive a $430 \mathrm{mg}$ TT softgel in the morning and another in the evening daily for 12 weeks.

\section{Dietary intake, physical activity, concomitant medication assessment and quality of life}

A food frequency questionnaire and a physical activity $\log$ will be collected at the baseline and 12-week visits to account for any drastic changes in the intake of macronutrients and micronutrients and deviations from the usual activities that could affect outcome measures. Prescription and over-the-counter medications and dietary supplement, as well as the reasons for taking these substances, will be recorded for the study period.

General health status will be measured with the Medical Outcomes Study 36-item short form Health Survey (SF-36, V.2) at baseline and 12 weeks of study. SF-36 has been reported to have good validity, internal consistency and reliability in the assessment of physical and mental health status of participants and their progression. ${ }^{50} 51$ The SF-36 consists of eight dimensions of health (physical function, bodily pain, general health, vitality, mental health, social function, and role of physical and emotional health) in the conduct of daily activity. ${ }^{52}$

\section{Blinding and unblinding}

Study participants and investigators including biostatistician, coordinators, and measurement and site personnel will be blinded to intervention allocation throughout this study. The investigators will be supplied with a blind code-breaker envelope for each participant. The blind code will not be broken except in a medical emergency or a potential study-related adverse event determined by the principal investigator. Medical emergencies may include abnormal elevation in liver function (ALT, AST) according to the regulation of FDA for TT intake.
Sample collection

Blood will be drawn between 8:00 and 10:00 from a superficial arm vein will be allowed to clot in a vacutainer at room temperature. Within 2 hours of collection, blood samples will be centrifuged at $1500 \times \mathrm{g}$ for $10 \mathrm{~min}$ and aliquoted. Urine samples will be collected in acidwashed polyethylene containers and aliquoted. Simultaneously collected blood and urine samples will be stored in $-80^{\circ} \mathrm{C}$ freezers prior to biochemical analyses for primary and secondary outcome measures.

\section{Outcomes measures}

In the present study, the primary outcome is urinary NTX. The secondary outcomes include serum BAP, sRANKL, OPG, TT and TP concentrations, urinary 8-OHdG, and quality of life. In addition, we will monitor adherence and compliance, adverse events, food intake and physical activity throughout the study period.

\section{Evaluation of adherence and compliance}

We will measure adherence to the intervention by counting the softgels consumed and determine compliance by the percentage of all softgels ingested. We will also measure serum concentrations of TT and TP at baseline and 12-week visit as additional evidence for adherence and compliance.

\section{Evaluation of adverse events}

Despite the GRAS reports that TT at the doses used in this study has minimal liver toxicity in humans, we will monitor the liver function by measuring AST and ALT at 0,6 and 12 weeks. Adverse effects associated with dietary supplement treatments, if any, will be self-reported by the participants and will be monitored by observing liver function every 6 weeks in the course of the intervention trial. All adverse events, whether observed by investigators or voluntarily disclosed by participants, will be recorded on the adverse event form throughout the study.

\section{Measurements}

Every participant will be evaluated at 0 (prior to starting intervention) 6 and 12 weeks of intervention.

\section{Bone biomarkers}

Rationale: Biochemical markers of bone turnover are promising in predicting fractures in the elderly for up to 2 years prior to the event. ${ }^{14}$ Serum BAP (a bone formation biomarker) and urinary NTX (a bone resorption biomarker) are more thorough clinical indicators of bone status than BMD in predicting skeletal response to a dietary supplement and in monitoring bone resorption changes as early as 3 months following initiation of intervention. ${ }^{53}$ On the other hand, studies have demonstrated that sRANKL and its decoy receptor OPG constitute a complex physiological mediator system involved in the regulation of bone resorption and may be responsible for the homoeostatic mechanism of normal bone 
remodelling. ${ }^{54}$ The serum BAP, RANKL, OPG ${ }^{55} 56$ and urine NTX $^{57} 58$ are commonly used by bone researchers to monitor the changes in bone remodelling due to treatments.

Methods: The serum concentration of bone formation biomarker, BAP, will be measured using Metra BAP immunoassay kits (Quidel Corporation, San Diego, California, USA); the intra-assay and interassay coefficient of variation (CVs) are $5.2 \%$ and $5.0 \%$, respectively. The concentration of bone resorption biomarker, NTX, in urine will be quantified using a commercial kit with a monoclonal antibody specific for the urinary $N$-terminal telopeptide (Alere, Providence, Rhode Island, USA). The intra-assay and interassay CVs are $2.2 \%$ and $3.0 \%$, respectively. The concentrations of creatinine in urine will be measured using MicroVue Creatinine Assay Kit (Quidel Corporation). The final NTX will be normalised by urinary creatinine. The serum concentration of sRANKL will be measured using sRANKL (total) human ELISA (BioVendor, LLC, Asheville, North Carolina, USA); the intra-assay and interassay CVs are $9.38 \%$ and $11.99 \%$, respectively. The serum concentration of OPG will be quantified using a commercial MicroVue OPG kit (Quidel Corporation). The intra-assay and interassay CVs are $2.8 \%$ and $5.1 \%$, respectively. In order to avoid the interassay variation, the samples from 0 -week, 6-week and 12-week visits of the same patients will be measured for bone biomarkers within the same assay each time.

\section{Urinary 8-OHdG level}

Rationale: Urinary 8-OHdG measurement provides a sensitive and non-invasive way to evaluate the efficacy of dietary antioxidant supplements, such as TT. reactive oxygen species (ROS)-mediated macromolecule oxidation produces $\mathrm{C} 8-\mathrm{OH}$-adduct radical with hydroxylation of guanine at the $\mathrm{C} 8$ position ${ }^{59} \mathrm{C} 8-\mathrm{OH}$-adduct radical is subsequently converted to 8-OH-Guanine (8-OH-Gua) by a one-electron oxidation. ${ }^{60}$ Unlike damaged lipids and proteins, impaired DNA cannot be removed by metabolic turnover of molecules; instead, damaged DNA has to be repaired in situ or destroyed by apoptotic processes to avoid mutations. In humans, 8-OH-Glu glycosylase performs a short-patch base-excision repair ${ }^{61}$ to remove 8-OH-Gua, which is later converted to 8-OHdG and excreted into urine without further metabolism. The stability of urinary 8-OHdG renders the molecule a putative biomarker for oxidative stress and DNA damage. $^{62}$

Methods: The serum concentrations of 8-OHdG will be measured by OxiSelect Oxidative DNA Damage ELISA Kit (Cell Biolabs, San Diego, California, USA) following the manufacture's instruction. The intra-assay and interassay CVs were $9.38 \%$ and $11.99 \%$, respectively.

\section{Serum TT and TP concentrations}

Rationale: The bioavailability and accumulation of TT is considered when we quantitatively evaluate the biological effects of TT intervention. Therefore, we will measure serum TT and TP concentrations of participants at 0,6 and 12 weeks.

Methods: Internal standard (IS) retinyl acetate will be dissolved into methanol. We will mix $500 \mu \mathrm{L}$ of serum and IS, then add $2 \mathrm{~mL}$ of $1 \%$ ascorbic acid in ethanol (wt/vol) and $25 \mu \mathrm{L}$ of butylated hydroxytoluene (BHT) $(10 \mathrm{mg} / 100 \mathrm{~mL})$, and $2 \mathrm{~mL}$ of $\mathrm{n}$-hexane. After vortex for $1 \mathrm{~min}$, the tube will be centrifuged at $1500 \times \mathrm{g}$ at $4^{\circ} \mathrm{C}$ for $5 \mathrm{~min}$ to facilitate phase separation. The supernatant will be transferred into a new tube. We will add additional $2 \mathrm{~mL}$ of $\mathrm{n}$-hexane into serum sample tube, and repeat vortex and centrifugation as described above. The supernatant will be transferred and combined with the previous extraction and will be dried under nitrogen. We will add methanol to the tube and transfer the extraction to an ultracentrifuge filter before the filtrate transfer to an high-performance liquid chromatography (HPLC) phial. Extracted samples will be stored at $-20^{\circ} \mathrm{C}$ prior to HPLC analysis. Serum concentrations of TT will be measured using a HPLC system (Waters Corporation) equipped with a Waters1525 binary pump, 2707 autosampler with refrigeration unit, 2489 dual wavelength ultraviolet-visible detector, 1525 multi $\lambda$ fluorescence detector and in-line degasser. The detection wavelength of TTs and TPs is $295 / 325 \mathrm{~nm}$, and wavelength for IS is $325 \mathrm{~nm}$. We will use a Phenomenex Kinetex PFP column $(2.6 \mu \mathrm{m}, 150 \times 4.6 \mathrm{~mm})$ with the mobile phase composed of methanol and water $(7: 1, \mathrm{v} / \mathrm{v})$ and a flow rate of $0.8 \mathrm{~mL} / \mathrm{min} .{ }^{63}$ We will measure the four isomers of TPs $(\alpha, \beta, \delta$ and $\gamma)$ and four isomers of TT $(\alpha, \beta, \delta$ and $\gamma)$ in sera of study participants.

\section{Statistical analysis}

In the study, the primary outcome is NTX at 12 weeks and intervention is TT at three levels: placebo, low dose and high dose. The outcome and other cofactors will be summarised using appropriate summary measures as per the type and distribution of the variable according to each intervention level. The baseline cofactors will be compared among the three groups using one-way ANOVA or Fisher's exact test $/ \chi^{2}$ test as appropriate.

To assess the effect of intervention on primary outcome (urine NTX) at 12 weeks, ANCOVA will be conducted after adjusting the baseline NTX and other significant cofactors, if any. A post hoc comparison among the three intervention groups will be made using Tukey's test if there is a significant effect of intervention. The assumptions of the ANCOVA model will be assessed and appropriate transformations will be made if needed for inferential data analysis. An 'intention-to-treat' analysis will be carried out for efficacious end point. The results will be summarised using mean differences and their 95\% CIs.

Similarly, the same analysis approach for NTX will be employed for serum BAP, RANKL, OPG, TTs and 8-OHdG. For secondary measures, the Pearson/ Spearman rank correlation between change in NTX and change in the secondary biomarker (BAP, TTs, RANKL, 
OPG, and 8-OHdG) will be computed and reported in tabular form. To evaluate quality of life, the ordinal score on the SF-36 test will be used, and therefore the Kruskal-Wallis test will be employed to detect any significant differences in the distribution of this score among the three groups followed by post hoc multiple comparisons if needed. $p$ Value $<5 \%$ will be considered as significant results. All the statistical analysis will be carried out using SAS V.9.3.

\section{Data management and data collection}

Researchers will keep information obtained in this study confidential except as required by law. All participant questionnaires, records and data will be coded and properly stored in locked cabinets. Only study investigators will have access to those files linking a person's study number to his/her name. Those forms will include the informed consent form, HIPAA authorisation form, all recruitment questionnaires and all other related materials.

The principle investigator will be responsible for oversight of data management of the trial. A certified monitor from Clinical Research Institute, independent from research team and sponsor, will assist the principle investigator in monitoring data collection before the data sets are delivered to study biostatistician. Questionnaire data will be entered on various forms, verified using computerised data entry forms and then entered into the Excel database. Laboratory data will also be verified and entered into an Excel database. Data of eligibility, medical records, attrition rate and compliance rate will all be entered into an Excel database. Data queries including missing values will be referred to the principle investigator. The principle investigator will have access to the final trial data set and disclosure of contractual agreements. The principle investigator will also incorporate any correction or addition into the datasets. Finally, a clean data set without any identification will be generated and delivered to the biostatistician for statistical analysis.

\section{Ethics}

An informed consent form will be signed by a participant before enrolling in the study. Any modifications to the protocol, which may affect the conduct of the study, potential benefits to the study participants, or their safety will be reported to the ethics committee for all necessary amendments. All study-related information will be stored securely at the study site in local cabinets, in an area with limited access (databases will be secured with a password-protected access system).

\section{Dissemination}

The findings of this trial will be submitted to a peerreviewed journal in the areas of nutrition or bone. Abstracts will be submitted to relevant national and international conferences.

\section{CONCLUSIONS}

The effectiveness of TT for bone health in postmenopausal women is still unknown. Our study, carried out at a research centre with experience in conducting independent, investigator-initiated FDA-IND clinical trial, intends to address a gap in the field and will test the safety and efficacy of TT supplementation for bone markers in postmenopausal women.

Author affiliations

${ }^{1}$ Department of Pathology, Texas Tech University Health Sciences Center, Lubbock, Texas, USA

${ }^{2}$ Department of Nutrition, Byrdine F. Lewis School of Nursing and Health Professions, Georgia State University, Atlanta, Georgia, USA

${ }^{3}$ Department of Nutritional Sciences, Texas Tech University, Lubbock, Texas, USA

${ }^{4}$ Department of Obstetrics and Gynecology, Texas Tech University Health Sciences Center, Lubbock, Texas, USA

${ }^{5}$ Department of Pharmacology, Universiti Kebangasaan Malaysia, Kuala Lumpur, Malaysia

Contributors C-LS conceptualised the study. C-LS, HM, SY, SW, CKF and INS designed this trial. C-LS developed the first draft of the manuscript and the rest of coauthors participated in the revision of subsequent drafts. All authors approved the final draft of the manuscript. C-LS made final decision to submit the report for publication.

Funding This study was supported by American River Nutrition, Hadley, Massachusetts, USA

Disclaimer The contents of this manuscript are solely the responsibility of the authors and do not necessarily represent the official views of American River Nutrition.

Competing interests This study is funded by American River Nutrition.

Patient consent Obtained.

Ethics approval The study received ethics approval from the Bioethics Committee of the Texas Tech University Health Sciences Center Institutional Review Board.

Provenance and peer review Not commissioned; externally peer reviewed.

Open Access This is an Open Access article distributed in accordance with the Creative Commons Attribution Non Commercial (CC BY-NC 4.0) license, which permits others to distribute, remix, adapt, build upon this work noncommercially, and license their derivative works on different terms, provided the original work is properly cited and the use is non-commercial. See: http:// creativecommons.org/licenses/by-nc/4.0/

\section{REFERENCES}

1. NIH. Consensus Development Panel on Osteoporosis Prevention, Diagnosis, and Therapy. Osteoporosis prevention, diagnosis, and therapy. JAMA 2001;285:785-95.

2. Looker AC, Melton LJ III, Harris TB, et al. Prevalence and trends in low femur bone density among older US adults: NHANES 20052006 compared with NHANES III. J Bone Miner Res 2010;25:64-71.

3. World Health Organization. Assessment of fracture risk and application to screening for postmenopausal osteoporosis. WHO Technical Report Series. Geneva, Switzerland: World Health Organization, 1994:843.

4. National Osteoporosis Foundation. American's bone health: the state of osteoporosis and low bone mass in our nation. Washington DC: National Osteoporosis Foundation, 2002:1-55.

5. Miller LJ III, Crowson CS, O'Fallon WM, et al. Relative contributions of bone density, bone turnover, and clinical risk factors to long-tern fracture prediction. J Bone Miner Res 2003;18:312-18.

6. Hui SL, Slemenda CW, Johnston CC Jr. Age and bone mass predictors of fracture in a prospective study. J Clin Invest 1988;81:1804-9.

7. Marshall D, Johnell O, Wedel H. Meta-analysis of how well measurements of bone mineral density predict the occurrence of osteoporotic fractures. BMJ 1996;312:1254-9. 
8. Vasikaran S, Eastell R, Bruyère O, et al. IOF-IFCC Bone Marker Standards Working Group: markers of bone turnover for the prediction of fracture risk and monitoring of osteoporosis treatment: a need for international reference standards. Osteoporos Int 2011;22:391-420

9. Worsfold M, Powell DE, Jones TJ, et al. Assessment of urinary bone markers for monitoring treatment of osteoporosis. Clin Chem 2004;50:2263-70.

10. Arjmandi BH, Khalil DA, Smith BJ, et al. Soy protein has a greater effect on bone in postmenopausal women not on hormone replacement therapy, as evidenced by reducing bone resorption and urinary calcium excretion. J Clin Endocrinol Metab 2003;88:1048-54.

11. Lucas EA, Wild RD, Hammond LJ, et al. Flaxseed improves lipid profile without altering biomarkers of bone metabolism in postmenopausal women. J Clin Endocrinol Metab 2002;87:1527-32.

12. Adachi JD. The correlation of bone mineral density and biochemical markers to fracture risk. Calcif Tissue Int 1996;59(Suppl I):S16-9.

13. Calvo MS, Eyre DR, Gundberg CM. Molecular basis and clinical application of biological markers of bone turnover. Endocr Rev 1996;17:333-68.

14. Lofman $\mathrm{O}$, Magnusson $\mathrm{P}$, Toss $\mathrm{G}$, et al. Common biochemical markers of bone turnover predict future bone loss: a 5-year follow-up study. Clin Chim Acta 2005;356:67-75.

15. Basu S, Michaëlsson $\mathrm{K}$, Olofsson $\mathrm{H}$, et al. Association between oxidative stress and bone mineral density. Biochem Biophys Res Commun 2001;288:275-9.

16. Banfi G, lorio EL, Corsi MM. Oxidative stress, free radicals and bone remodeling. Clin Chem Lab Med 2008;46:1550-5.

17. Ha H, Lee JH, Kim HN, et al. Alpha-tocotrienol inhibits osteoclastic bone resorption by suppressing RANKL expression and signaling and bone resorbing activity. Biochem Biophys Res Commun 2011;406:546-51

18. Nizar AM, Nazrun AS, Norazlina M, et al. Low dose of tocotrienols protects osteoblasts against oxidative stress. Clin Ter 2011:162:533-8.

19. Brooks R, Kalia P, Ireland DC, et al. Direct inhibition of osteoclast formation and activity by the vitamin $\mathrm{E}$ isomer gamma-tocotrienol. Int J Vitam Nutr Res 2011;81:358-67.

20. Abd Manan N, Mohamed N, Shuid AN. Effects of low-dose versus high-dose $\gamma$-tocotrienol on the bone cells exposed to the hydrogen peroxide-induced oxidative stress and apoptosis. Evid Based Complement Alternat Med 2012;2012:680834.

21. Abdul-Majeed S, Mohamed N, Soelaiman IN. The use of delta-tocotrienol and lovastatin for anti-osteoporotic therapy. Life Sci 2015; $125: 42-8$.

22. Ima-Nirwana S, Suhaniza S. Effects of tocopherols and tocotrienols on body composition and bone calcium content in adrenalectomized rats replaced with dexamethasone. J Med Food 2004;7:45-51.

23. Ahmad NS, Khalid BA, Luke DA, et al. Tocotrienol offers better protection than tocopherol from free radical-induced damage of rat bone. Clin Exp Pharmacol Physiol 2005;32:761-70.

24. Norazlina M, Ng FW, Ima-Nirwana S. $\gamma$-tocotrienol is required for normal vitamin D metabolism in female rats. Indian $\mathrm{J}$ Pharmacol 2005:37:309-14.

25. Hermizi $\mathrm{H}$, Faizah $\mathrm{O}$, Ima-Nirwana $\mathrm{S}$, et al. Beneficial effects of tocotrienol and tocopherol on bone histomorphometric parameters in sprague-dawley male rats after nicotine cessation. Calcif Tissue Int 2009;84:65-74.

26. Mehat MZ, Shuid AN, Mohamed N, et al. Beneficial effects of vitamin $\mathrm{E}$ isomer supplementation on static and dynamic bone histomorphometry parameters in normal male rats. $J$ Bone Miner Metab 2010;28:503-9.

27. Mohamad S, Shuid AN, Mokhtar SA, et al. Tocotrienol supplementation improves late-phase fracture healing compared to alpha-tocopherol in a rat model of postmenopausal osteoporosis: a biomechanical evaluation. Evid Based Complement Alternat Med 2012;2012:372878.

28. Norazlina $\mathrm{M}$, Hermizi $\mathrm{H}$, Faizah $\mathrm{O}$, et al. Vitamin $\mathrm{E}$ reversed nicotine-induced toxic effects on bone biochemical markers in Male rats. Arch Med Sci 2010;6:505-12.

29. Chin KY, Abdul-Majeed S, Fozi NF, et al. Annatto tocotrienol improves indices of bone static histomorphometry in osteoporosis due to testosterone deficiency in rats. Nutrients 2014;6:4974-83.

30. Abdul-Majeed S, Mohamed N, Soelaiman IN. Effects of tocotrieno and lovastatin combination on osteoblast and osteoclast activity in estrogen-deficient osteoporosis. Evid Based Complement Alternat Med 2012;2012:960742.

31. Chin KY, Ima-Nirwana S. Effects of annatto-derived tocotrienol supplementation on osteoporosis induced by testosterone deficiency in rats. Clin Interv Aging 2014;9:1247-59.
32. Muhammad N, Luke DA, Shuid AN, et al. Tocotrienol supplementation in postmenopausal osteoporosis: evidence from a laboratory study. Clinics (Sao Paulo). 2013;68:1338-43.

33. Muhammad N, Luke DA, Shuid AN, et al. Two different isomers of vitamin e prevent bone loss in postmenopausal osteoporosis rat model. Evid Based Complement Alternat Med 2012;2012:161527.

34. Maggio D, Barabani M, Pierandrei M, et al. Marked decrease in plasma antioxidants in aged osteoporotic women: results of a cross-sectional study. J Clin Endocrinol Metab 2003;88:1523-7.

35. Michaëlsson K, Wolk A, Byberg L, et al. Intake and serum concentrations of $\alpha$-tocopherol in relation to fractures in elderly women and men: 2 cohort studies. Am J Clin Nutr 2014;99:107-14.

36. Mata-Granados JM, Cuenca-Acebedo R, Luque de Castro MD, et al Lower vitamin E serum levels are associated with osteoporosis in early postmenopausal women: a cross-sectional study. J Bone Miner Metab 2013;31:455-60.

37. Ruiz-Ramos M, Vargas LA, Fortoul, et al. Supplementation of ascorbic acid and alpha-tocopherol is useful to preventing bone loss linked to oxidative stress in elderly. $J$ Nutr Health Aging 2010;14:467-72.

38. Hamidi MS, Corey PN, Cheung AM. Effects of vitamin E on bone turnover markers among us postmenopausal women. J Bone Miner Res 2012;27:1368-80.

39. Wolf RL, Cauley JA, Pettinger M, et al. Lack of a relation between vitamin and mineral antioxidants and bone mineral density: results from the Women's Health Initiative. Am J Clin Nutr 2005;82:581-8.

40. Ochi H, Takeda S. The two sides of vitamin E supplementation. Gerontology 2015;61:319-26.

41. Garrett IR, Boyce BF, Oreffo ROC, et al. Oxygen-derived free radicals stimulate osteoclastic bone resorption in rodent bone in vitro and in vivo. J Clin Invest 1990;85:632-9.

42. Dreher I, Schuetze N, Baur A, et al. Selenoproteins are expressed in fetal human osteoblast-like cells. Biochem Biophys Res Commun 1998;245:101-7.

43. Hopewell S, Clarke M, Moher D, et al., CONSORT Group. CONSORT for reporting randomized controlled trials in journal and conference abstracts: explanation and elaboration. PLOS Med 2008;5:e20.

44. Iwamoto J, Takada T. Effect of minodronate on the speed of sound of the calcaneus in postmenopausal women with an increased risk of fractures: a clinical practice-based observational study. J Chin Med Assoc 2015;78:591-6.

45. Kanders B, Dempster DW, Lindsay R. Interaction of calcium nutrition and physical activity on bone mass in young women. $J$ Bone Miner Res 1988;3:145-9.

46. Jackson LW, Cromer BA, Panneerselvamm A. Association between bone turnover, micronutrient intake, and blood lead levels in preand postmenopausal women, NHANES 1999-2002. Environ Health Perspect 2010;118:1590-6.

47. Shen CL, Chyu MC, Yeh JK, et al. Effect of green tea and Tai Chi on bone health in postmenopausal osteopenic women: a 6-month randomized placebo-controlled trial. Osteoporos Int 2012:23:1541-52.

48. Reagan-Shaw S, Nihal M, Ahmad N. Dose translation from animal to human studies revisited. FASEB J 2008:22:659-61.

49. Mahipal A, Klapman J, Vignesh S, et al. Pharmacokinetics and safety of vitamin $E$-tocotrienol after single and multiple doses in healthy subjects with measurement of vitamin E metabolites. Cancer Chemother Pharmacol 2016;78:157-65.

50. Ware J Jr, Kosinski M, Keller SD. A 12-item Short-Form Health Survey: construction of scales and preliminary tests of reliability and validity. Med Care 1996;34:220-33.

51. Pickard AS, Johnson JA, Penn A, et al. Replicability of SF-36 summary scores by the SF-12 in stroke patients. Stroke 1999;30:1213-7.

52. Ware J, Snow KK, Kosinski M. SF-36 health survey manual and interpretation guide. Boston, MA: Health Institute, New England Medical Center Hospital, 1993.

53. Yamazaki S, Ichimura S, Iwamoto J, et al. Effect of walking exercise on bone metabolism in postmenopausal women with osteopenia/ osteoporosis. J Bone Miner Metab 2004;22:500-8.

54. Rogers A, Eastell R. Circulating osteoprotegerin and receptor activator for nuclear factor kappaB ligand: clinical utility in metabolic bone disease assessment. J Clin Endocrinol Metab 2005:90:6323-31.

55. Marques EA, Mota J, Viana JL, et al. Response of bone mineral density, inflammatory cytokines, and biochemical bone markers to a 32-week combined loading exercise programme in older men and women. Arch Gerontol Geriatr 2013;57:226-33.

56. Josse AR, Atkinson SA, Tarnopolsky MA, et al. Diets higher in dairy foods and dietary protein support bone health during diet- and 
exercise-induced weight loss in overweight and obese premenopausal women. J Clin Endocrinol Metab 2012;97:251-60.

57. Dawson-Hughes B, Harris SS, Palermo NJ, et al. Potassium bicarbonate supplementation lowers bone turnover and calcium excretion in older men and women: a randomized dose-finding trial. $J$ Bone Miner Res 2015;30:2103-11.

58. Kanellakis S, Moschonis G, Tenta R, et al. Changes in parameters of bone metabolism in postmenopausal women following a 12-month intervention period using dairy products enriched with calcium, vitamin $\mathrm{D}$, and phylloquinone (vitamin $\mathrm{K}(1))$ or menaquinone-7 (vitamin K (2)): the Postmenopausal Health Study II. Calcif Tissue Int 2012;90:251-62.

59. Steenken S. Purine bases, nucleosides, and nucleotides: aqueous solution redox chemistry and transformation reactions of their radical cations and e-and OH adduct. Chem Rev 1989;89:503-20.
60. Kasai H, Yamaizumi Z, Yamamoto F, et al. Photosensitized formation of 8-hydroxyguanine (7,8-dihydro-8-oxoguanine) in DNA by riboflavin. Nucleic Acids Symp Ser 1992;27: 181-2.

61. Evans MD, Dizdaroglu M, Cooke MS. Oxidative DNA damage and disease: induction, repair and significance. Mutat Res 2004:567:1-61.

62. Pilger A, Germadnik D, Riedel K, et al. Longitudinal study of urinary 8-hydroxy-2-deoxyguanosine excretion in healthy adults. Free Radic Res 2001;35:273-80.

63. Grebenstein N, Frank J. Rapid baseline-separation of all eight tocopherols and tocotrienols by reversed-phase liquid-chromatography with a solid-core pentafluorophenyl column and their sensitive quantification in plasma and liver. $J$ Chromatogr $A$ 2012;1243:39-46. 